Revista de Matemática: Teoría y Aplicaciones 4(2): 13-23 (1997)

\title{
THE HANKEL TRANSFORM OF CAUSAL DISTRIBUTIONS
}

\author{
Manuel A. Aguirre ${ }^{1}$
}

\begin{abstract}
In this note we evaluate the unidimensional distributional Hankel transform of $\frac{x_{+}^{\alpha-1}}{\Gamma^{(\alpha)}}, \frac{x_{-}^{\alpha-1}}{\Gamma^{(\alpha)}}, \frac{|x|^{\alpha-1}}{\Gamma^{(\alpha / 2)}}, \frac{|x|^{\alpha-1} \operatorname{sgn}(x)}{\Gamma^{\left(\frac{\alpha+1}{2}\right)}}$ and $(x \pm i 0)^{\alpha-1}$ and then we extend the formulae to certain kinds of $n$-dimensional distributions called "causal" and "anti-causal" distributions. We evaluate the distributional Hankel transform of $\frac{\left(m^{2}+P\right)_{+}^{\alpha-1}}{\Gamma^{(\alpha)}}$, $\frac{\left(m^{2}+P\right)_{-}^{\alpha-1}}{\Gamma^{(\alpha)}}, \frac{\left|m^{2}+P\right|^{\alpha-1}}{\Gamma\left(\frac{\alpha}{2}\right)}, \frac{\left|m^{2}+P\right|^{\alpha-1} \operatorname{sgn}\left(m^{2}+P\right)}{\Gamma\left(\frac{\alpha+1}{2}\right)}$ and $\left(m^{2}+P \pm i 0\right)^{\alpha-1}$.
\end{abstract}

\section{Introduction}

Let $U(t) \in S_{R^{+}}^{\prime}$, where $S_{R^{+}}^{\prime}$ is the dual of $S_{R^{+}}\left(S_{R^{+}}\right.$is the space of functions $f \in S$ defined in $\left.R^{+}=\{t: t>0\}\right)$.

The Hankel transform of $U(t)$ will be, by definition, the distribution $V(S) \in S_{R^{+}}^{\prime}$ defined by the formula

$$
\langle H\{U(t)\}, \varphi(s)\rangle=\langle U(t), H\{\varphi(s)\}\rangle,
$$

for every $\varphi \in S_{R^{+}}$(cf. [6], page 26, formula $(\mathrm{I}, 5,6)$ ).

By the Hankel transform of the function $f(x)$ we mean the function $g(s), 0 \leq s<\infty$, defined by the formula

$$
g(s)=(H\{f(t)\})=\frac{1}{2} \int_{0}^{\infty} f(t) t^{\frac{n-2}{2}} R_{\frac{n-2}{2}}(\sqrt{t s}) d t
$$

where

$$
R_{\gamma}(x)=\frac{J_{\gamma}(x)}{x^{\gamma}}
$$

and

$$
J_{\gamma}(x)=\sum_{\nu=0}^{\infty} \frac{(-1)^{\nu}\left(\frac{x}{2}\right)^{\gamma+2 \nu}}{\nu ! \Gamma(\gamma+\nu+1)}
$$

\footnotetext{
${ }^{1}$ Facultad de Ciencias Exactas de la Universidad Nacional del Centro de la Provincia de Buenos Aires, Pinto 399, 3er. Piso, 7000 Tandil, Argentina.
} 
Similarly, let $U(t) \in S_{R^{-}}^{\prime}$. The Hankel transform of $U(t)$ will be, by definition, the distribution $V(s) \in S_{R^{-}}^{\prime}$, defined by the formula:

$$
\langle H\{U(t)\}, \varphi(s)\rangle=\langle U(t), H\{\varphi(s)\}\rangle
$$

for every $\varphi \in S_{R^{-}}$. Here $S_{R^{-}}^{\prime}$ designates the dual of $S_{R^{-}}$.

By $S_{R^{-}}$we designate the space of functions $f \in S$ defined in the negative half line $R^{-}=\{t: t<0\}$. By the Hankel transform of the function $\varphi(s)$ we mean the function $h(t),-\infty<t<0$, defined by the formula:

$$
h(t)=H\{\varphi(s)\}=(-1) \frac{1}{2} \int_{-\infty}^{0} \varphi(s) S^{\frac{n-2}{2}}(\sqrt{s t}) d s
$$

Let $\phi_{s}$ be a distribution of variable $s$, and let $U(x) \in C^{\infty}\left(R^{n}\right)$ be such that the (n-1)-dimensional manifold $U\left(x_{1}, \ldots, x_{n}\right)=0$ has no critical points; $\varphi_{u(x)}$ denotes the distribution defined on $R^{n}$ be the formula (called the Leray formula ([2], p. 102)

$$
\int_{R^{n}} \phi_{u(x)} f(x) d x_{1} \ldots d x_{n}=\int_{-\infty}^{\infty} \phi_{s} d_{s} \int_{u(x)=s} f(x) W_{n}(x, d x) ;
$$

here $w_{n}$ is an (n-1)-dimensional form on $u$ defined as follows:

$$
d u \wedge d w=d x_{1} \wedge d x_{2} \wedge \ldots \wedge d x_{n}
$$

the manifold $u(x)=s$ has the orientation such that $W_{n}(x, d x)>0$.

On the other hand, from [5], p. 2, formula (11) we have,

$$
\langle H\{\Phi(U(x))\}, \varphi\rangle=\langle H\{V(t)\}, \varphi(s)\rangle
$$

where

$$
V(t)=\Phi(U)
$$

and

$$
\psi(s)=\int_{U=S} \varphi W(x, d x)
$$

In this note we shall evaluate the unidimensional distributional Hankel transform of $\frac{x_{+}^{\alpha-1}}{\Gamma(\alpha)}, \frac{x_{-}^{\alpha-1}}{\Gamma(\alpha)}, \frac{|x|^{\alpha-1}}{\Gamma\left(\frac{\alpha}{2}\right)}, \frac{|x|^{\alpha-1} \operatorname{sgn}(x)}{\Gamma\left(\frac{\alpha+1}{2}\right)}$, and $(x \pm i 0)^{\alpha-1}$ (cf. formulae (32), (34), (36), (37), (41) and (42) and then we extend the formulae to distributions called "causal" and "anticausal" distribution. We evaluate the distributional Hankel transform of $\frac{\left(m^{2}+P\right)_{+}^{\alpha-1}}{\Gamma(\alpha)}$, $\frac{\left(m^{2}+P\right)_{-}^{\alpha-1}}{\Gamma(\alpha)}, \frac{\left(m^{2}+P\right)^{\alpha-1}}{\Gamma\left(\frac{\alpha}{2}\right)}, \frac{\left(m^{2}+P\right)^{\alpha-1} \operatorname{sgn}\left(m^{2}+P\right)}{\Gamma\left(\frac{\alpha+1}{2}\right)}$ and $\left(m^{2}+P \pm i 0\right)^{\alpha-1}$ (cf. formulae (72), (75), (78), (81) and (83)). 
2 The distributional Hankel transform of $\frac{x_{+}^{\alpha-1}}{\Gamma(\alpha)}, \frac{x_{=}^{\alpha-1}}{\Gamma(\alpha)}, \frac{x^{\alpha-1}}{\Gamma\left(\frac{\alpha}{2}\right)}$, $\frac{|x|^{\alpha-1} \operatorname{sgn}(x)}{\Gamma\left(\frac{\alpha-1}{2}\right)}$, and $(x \pm i 0)^{\alpha-1}$.

Consider the family of generalized functions defined by the formulae,

$$
\begin{aligned}
R_{\alpha}^{+}(x) & =\frac{x_{+}^{\alpha-1}}{\Gamma(\alpha)} \\
R_{\alpha}^{-}(x) & =\frac{x_{-}^{\alpha-1}}{\Gamma(\alpha)} \\
Y_{\alpha}(x) & =\frac{|x|^{\alpha-1}}{\Gamma\left(\frac{\alpha}{2}\right)}
\end{aligned}
$$

and

$$
Z_{\alpha}(x)=\frac{|x|^{\alpha-1} \operatorname{sgn}(x)}{\Gamma\left(\frac{\alpha-1}{2}\right)} .
$$

Let $f_{\alpha}(x \pm i 0)$ be a function defined by

$$
f_{\alpha}(x \pm i 0)=(x \pm i 0)^{\alpha-1}=x_{+}^{\alpha-1}+e^{ \pm(\alpha-1) \pi i)} x_{-}^{\alpha-1}
$$

where,

$$
\begin{gathered}
x_{+}^{\lambda}=\left\{\begin{array}{ll}
x^{\lambda} & \text { for } x>0 \\
0 & \text { for } x \leq 0
\end{array},\right. \\
x_{-}^{\lambda}=\left\{\begin{array}{ll}
|x|^{\lambda} & \text { for } x<0 \\
0 & \text { for } x \geq 0
\end{array},\right. \\
|x|^{\lambda}=x_{+}^{\lambda}+x_{-}^{\lambda} \\
|x|^{\lambda} \operatorname{sgn}(x)=x_{+}^{\lambda}-x_{-}^{\lambda} \\
(x \pm i 0)^{\lambda}=\lim _{\varepsilon \rightarrow 0}(x \pm i \varepsilon)^{\lambda}
\end{gathered}
$$

$\lambda$ is a complex number and

$$
\operatorname{sgn}(x)=\left\{\begin{array}{ll}
1 & \text { if } x>0 \\
-1 & \text { if } x<0
\end{array} .\right.
$$

Gelfand and Shilov ([3], p. 48-60) have studied in detail the functions (16), (17), (18), (19) and (20). These functions are locally integrables and define generalized functions for all $\lambda \neq-1,-2, \ldots$; except (20) which is an entire function of $\lambda$.

The distributions (16), (17), (18) and (19) are analytic in $\lambda$ except at $\lambda=-1,-2, \ldots$, where they have simple poles, therefore from [3], pag. 57-58 and taking into account the formula,

$$
\operatorname{Res}_{\alpha=-k} \Gamma(\alpha)=\frac{(-1)^{k}}{k !}
$$


we have

$$
\begin{aligned}
\lim _{\alpha \rightarrow-k} R_{\alpha}^{+}(x) & =\delta^{(k)}(x), \\
\lim _{\alpha \rightarrow-k} R_{\alpha}^{-}(x) & =(-1)^{k} \delta^{(k)}(x), \\
\lim _{\alpha \rightarrow-2 k} Y_{\alpha}(x) & =\frac{(-1)^{k} k !}{(2 k) !} \delta^{(2 k)}(x),
\end{aligned}
$$

and

$$
\lim _{\alpha \rightarrow-1 k-1} Z_{\alpha}(x)=\frac{(-1)^{k+1} k !}{(2 k+1) !} \delta^{(2 k+1)}(x)
$$

where

$$
\Gamma(\alpha)=\int_{0}^{\infty} e^{-x} x^{\alpha-1} d x
$$

By taking into account the formula,

$$
\int_{0}^{\infty} x^{u} J_{\nu}(a x) d x=\frac{2^{u} a^{-u-1} \Gamma\left(\frac{1}{2}+\frac{\nu}{2}+\frac{\mu}{2}\right)}{\Gamma\left(\frac{1}{2}+\frac{\nu}{2}+\frac{\mu}{2}\right)}
$$

- Real $\nu-1<$ Real $\mu<\frac{1}{2}, a>0$ ([9], page 684 , formula 14).

From the formulae (1) and (5) we shall get the Hankel distributional transform of the family of generalized functions defined by the formulae (11), (12), (13), (14) and (15).

In fact, from (1) and (2), we have,

$$
\begin{aligned}
\left\langle H\left\{R_{\alpha}^{+}(t)\right\}, \varphi\right\rangle=\left\langle H\left[\frac{t_{+}^{\alpha-1}}{\Gamma(\alpha)}\right], \varphi\right\rangle= & \\
= & \left\langle\frac{t_{+}^{\alpha-1}}{\Gamma(\alpha)}, H[\varphi(s)]\right\rangle=\frac{1}{\Gamma(\alpha)} \int_{0}^{\infty} t^{\alpha-1} H[\varphi(s)] d t= \\
= & \left\langle\frac{1}{2} S^{\frac{n-2}{2}} \frac{1}{\Gamma(\alpha)} \int_{0}^{\infty} t^{\alpha-1} R_{\frac{n-2}{2}}(\sqrt{s t}) d t, \varphi(s)\right\rangle \cdot(29)
\end{aligned}
$$

On the other hand, taking into account the formulae (3) and (28) we have,

$$
\begin{aligned}
& \frac{1}{2} \frac{1}{\Gamma(\alpha)} \int_{0}^{\infty} t^{\alpha-1} R_{\frac{n-2}{2}}(\sqrt{s t}) d t= \\
= & \frac{1}{\Gamma(\alpha)} \int_{0}^{\infty} t^{2 \alpha-\frac{n}{2}} S^{-\frac{n}{4}+\frac{1}{2}} J_{\frac{n-2}{2}}(\sqrt{s} \cdot t) d t= \\
= & \frac{S^{-\frac{n}{4}+\frac{1}{2}}}{\Gamma(\alpha)}\left[\frac{2^{2 \alpha-\frac{n}{2}} S^{-\alpha+\frac{n}{4}+\frac{1}{2}} \Gamma(\alpha)}{\Gamma\left(\frac{n}{2}-\alpha\right)}\right]= \\
= & \frac{S^{-\alpha}}{2^{\frac{n}{2}-2 \alpha} \Gamma\left(\frac{n}{2}-\alpha\right)} .
\end{aligned}
$$

Therefore, from (29) and taking into account the formulae (30) and (31) we have,

$$
\left\langle H\left\{R_{\alpha}^{+}(t)\right\}, \varphi\right\rangle=\frac{1}{2^{\frac{n}{2}-2 \alpha}}\left\langle R_{\frac{n}{2}-\alpha}^{+}(s), \varphi\right\rangle .
$$


From (31) we obtain the following formula:

$$
H\left\{R_{\alpha}^{+}(t)\right\}=\frac{1}{2^{\frac{n}{2}-2 \alpha}} R_{\frac{n}{2}-\alpha}^{+}(s)
$$

Similarly, from (5) and (6) we have,

$$
\begin{aligned}
& \left\langle H\left\{R_{\alpha}^{-}(t)\right\}, \varphi(s)\right\rangle=\left\langle R_{\alpha}^{-}(t), H\{\varphi(s)\}\right\rangle= \\
= & \int_{-\infty}^{0} \frac{|t|^{\alpha-1}}{\Gamma(\alpha)} H\{\varphi(s)\} d t= \\
= & \frac{1}{\Gamma(\alpha)} \int_{0}^{\infty} t^{\alpha-1}\left\{(-1) \frac{1}{2} \int_{-\infty}^{0} \varphi(s) S^{\frac{n-2}{2}} R_{\frac{n-2}{2}}(\sqrt{s t}) d s\right\} d t= \\
= & \frac{1}{\Gamma(\alpha)} \frac{(-1)}{2}\left\langle(-S)^{\frac{n-2}{2}} \int_{0}^{\infty} t^{\alpha-1} R_{\frac{n-2}{2}}(\sqrt{s t}) d t, \varphi(-s)\right\rangle .
\end{aligned}
$$

Taking into account the formula (30) and the formula (12), from (33) we have,

$$
\begin{aligned}
& \left\langle H\left\{R_{\alpha}^{-}(t)\right\}, \varphi(s)\right\rangle=\frac{(-1)^{\frac{n}{2}}}{2^{\frac{n}{2}-2 \alpha}}\left\langle\frac{S^{-\alpha+\frac{n-2}{2}}}{\Gamma\left(\frac{n}{2}-\alpha\right)}, \varphi(s)\right\rangle= \\
& =\left\langle\frac{(-1)^{\frac{n}{2}}}{2^{\frac{n}{2}-2 \alpha}} R_{\frac{n}{2}-\alpha}^{-}(s), \varphi(s)\right\rangle,
\end{aligned}
$$

therefore,

$$
H\left\{R_{\alpha}^{-}(t)\right\}=\frac{(-1)^{\frac{n}{2}}}{2^{\frac{n}{2}-2 \alpha}} R_{\frac{n}{2}-\alpha}^{-}(s)
$$

On the other hand, the family of generalized functions defined by the formulae (13) and (14) are even and odd combinations of $R_{\alpha}^{+}(t)$ and $R_{\alpha}^{-}(t)$, therefore to obtain its Hankel transform we take into account the results (32) and (34). In fact, from (13), (18), (32), (34) and the Lagrange's duplication formula

$$
\Gamma(2 Z)=2^{2 Z-1} \pi^{-\frac{1}{2}} \Gamma(Z) \Gamma\left(Z+\frac{1}{2}\right)
$$

([8], page 344, formula 15), we have

$$
\begin{gathered}
H\left\{Y_{\alpha}(t)\right\}=H\left\{\frac{|t|^{\alpha-1}}{\Gamma\left(\frac{\alpha}{2}\right)}\right\}= \\
=\frac{\Gamma\left(\frac{\alpha+1}{2}\right)}{2^{\frac{n}{2}-3 \alpha+1} \pi^{\frac{1}{2}}}\left[R_{\frac{n}{2}-\alpha}^{+}(s)+(-1)^{\frac{n}{2}} R_{\frac{n}{2}-\alpha}^{-}(s)\right]
\end{gathered}
$$

Similary, from (14), (19), (32), (34) and taking into account the formula (35), we have,

$$
\begin{gathered}
H\left\{Z_{\alpha}(t)\right\}=H\left\{\frac{|t|^{\alpha-1} \operatorname{sgn}(t)}{\Gamma\left(\frac{\alpha+1}{2}\right)}\right\}= \\
=\frac{\Gamma\left(\frac{\alpha}{2}\right)}{2^{\frac{n}{2}-3 \alpha+1} \pi^{\frac{1}{2}}}\left[R_{\frac{n}{2}-\alpha}^{+}(s)-(-1)^{\frac{n}{2}} R_{\frac{n}{2}-\alpha}^{-}(s)\right] .
\end{gathered}
$$


Finally to obtain the Hankel transform of $f_{\alpha}(x \pm i 0)$ we shall take into account the results (32) and (34).

In fact, for $n$ even, from (15) and taking into account the formulae (11), (12), (32) and (34) we have,

$$
\begin{gathered}
H\left\{f_{\alpha}(t+i 0)\right\}=H\left\{t_{+}^{\alpha-1}\right\}+e^{(\alpha-1) \pi i} H\left\{t_{-}^{\alpha-1}\right\}= \\
=\Gamma(\alpha)\left[H\left\{R_{\alpha}^{+}(t)\right\}+e^{(\alpha-1) \pi i} H\left\{R_{\alpha}^{-}(t)\right\}\right]= \\
=\frac{\Gamma(\alpha)}{2^{\frac{n}{2}-2 \alpha}}\left[\frac{S_{+}^{\frac{n}{2}-\alpha-1}}{\Gamma\left(\frac{n}{2}-\alpha\right)}+e^{\alpha-1) \pi i}(-1)^{\frac{n}{2}} \frac{S_{-}^{\frac{n}{2}-\alpha-1}}{\Gamma\left(\frac{n}{2}-\alpha\right)}\right]= \\
=\frac{\Gamma(\alpha)}{2^{\frac{n}{2}-2 \alpha} \Gamma\left(\frac{n}{2}-\alpha\right)}(S-i 0)^{\frac{n}{2}-\alpha-1},
\end{gathered}
$$

for $\alpha \neq-k, k=0,1,2, \ldots$

Similarly, for $n$ even and $\alpha \neq-k, k=0,1,2, \ldots$, we have

$$
\begin{gathered}
H\left\{f_{\alpha}(t-i 0)\right\}=H\left\{(t-i 0)^{\alpha-1}\right\}= \\
=\frac{\Gamma(\alpha)}{2^{\frac{n}{2}-2 \alpha} \Gamma\left(\frac{n}{2}-\alpha\right)}(S+i 0)^{\frac{n}{2}-\alpha-1} .
\end{gathered}
$$

In particular, for the case $\alpha=-k, k=0,1,2, \ldots$, and taking into account the formula (23) and (32) we get the following result,

$$
H\left\{\delta^{(k)}(t)\right\}=\frac{1}{2^{\frac{n}{2}+2 k}} R_{\frac{n}{2}+k}^{+}(s)=\frac{1}{2^{\frac{n}{2}+2 k} \Gamma\left(\frac{n}{2}+k\right)} S_{+}^{\frac{n-2}{2}+k} .
$$

The formula (43) was proved in [6], page 28 , formula $(\mathrm{I}, 5,15)$ by S.E. Trione.

We observe that if we consider the following family of generalized functions $N_{\alpha}(x \pm i 0)$ defined by the formula

$$
N_{\alpha}(x \pm i 0)=\frac{f_{\alpha}(x \pm i 0)}{\Gamma(\alpha)}
$$

where $f_{\alpha}(x \pm i 0)$ is defined by (15) and taking into account the formulae (41) and (42), then the Hankel distributional transform of $N_{\alpha}(x \pm i 0)$ can be expressed by the formula,

$$
H\left\{N_{\alpha}(t \pm i 0)\right\}=\frac{1}{2^{\frac{n}{2}-2 \alpha}} N_{\frac{n}{2}-\alpha}(S \mp i 0)
$$

for $n$ even and $\alpha \neq-k, k=0,1,2, \ldots$

On the other hand, the generalized function $(x \pm i 0)^{\lambda}$ are entire of $\lambda([3]$, page 60$)$.

In the points $\lambda=-k, k=1,2, \ldots$, from [3], page 94 , the following formula is valid,

$$
(x \pm i 0)^{-k}=x^{-k} \mp \frac{\pi i(-1)^{k-1}}{(k-1) !} \delta^{(k-1)}(x),
$$

therefore for the case $\alpha=-k, k=0,1,2, \ldots$ taking into account the formulae (23) and (24) we have,

$$
\lim _{\alpha \rightarrow-k} N_{\alpha}(x \pm i 0)=0
$$

where the limit is in the sense of generalized functions. 


\section{The distributional Hankel transform of $\frac{\left(m^{2}+P\right)_{+}^{\alpha=1}}{\Gamma(\alpha)}, \frac{\left(m^{2}+P\right)_{\underline{\alpha}}^{\alpha=1}}{\Gamma(\alpha)}$, $\frac{\left|m^{2}+P\right|^{\alpha-1}}{\Gamma\left(\frac{\alpha}{2}\right)}, \frac{\left|m^{2}+P\right|^{\alpha-1} \operatorname{sgn}\left(m^{2}+P\right)}{\Gamma\left(\frac{\alpha+1}{2}\right)}$ and $\left(m^{2}+P \pm i 0\right)^{\alpha=1}$.}

In this paragraph we are going to extend the results (32), (34), (36), (37), (41) and (42) to certain kinds of $n$-dimensional distributions called "causal" and "anticausal" distributions.

We begin with some definitions. Let $x=\left(x_{1}, x_{2}, \ldots, x_{n}\right)$ be a point of the $n$-dimensional euclidean space $R^{n}$. Consider a non degenerate quadratic form in $n$ variables of the form

$$
P=P(x)=x_{1}^{2}+\cdots+x_{\mu}^{2}-x_{\mu+1}^{2}-\cdots-x_{\mu+\nu}^{2},
$$

where $\mu+\nu=n$ (dimension of the space).

The distribution $(P \pm i 0)^{\lambda}$ are defined by

$$
(P \pm i 0)^{\lambda}=\lim _{\varepsilon \rightarrow 0}\left(P \pm i \varepsilon|x|^{2}\right)^{\lambda},
$$

where

$$
\varepsilon>0,|x|^{2}=x_{1}^{2}+\cdots+x_{n}^{2}
$$

and $\lambda$ complex number.

The distributions $\left(m^{2}+P \pm i 0\right)^{\lambda}$ are defined in an analogue manner as the distribution $(P \pm i 0)^{\lambda}$. Let us put (cf. [3], page 289)

$$
\left(m^{2}+P \pm i 0\right)^{\lambda}=\lim _{\varepsilon \rightarrow 0}\left(m^{2}+P \pm i 0|x|^{2}\right)^{\lambda},
$$

where $\varepsilon$ is an arbitrary positive number and $m$ is positive real number.

It is useful to state an equivalent definition of the distribution $\left(m^{2}+P \pm i 0\right)^{\lambda}$. In this definition appear the distributions

$$
\left(m^{2}+P\right)_{+}^{\lambda}= \begin{cases}\left(m^{2}+P\right)^{\lambda} & \text { if }\left(m^{2}+P\right) \geq 0 \\ 0 & \text { if } m^{2}+P<0\end{cases}
$$

and

$$
\left(m^{2}+P\right)_{-}^{\lambda}=\left\{\begin{array}{ll}
0 & \text { if } m^{2}+P>0 \\
\left(-m^{2}-P\right)^{\lambda} & \text { if } m^{2}+P \leq 0
\end{array} .\right.
$$

From [4], page 566, we have

$$
f_{\lambda}\left(m^{2}+P \pm i 0\right)=\left(m^{2}+P\right)_{+}^{\lambda}+e^{ \pm \lambda \pi i}\left(m^{2}+P\right)_{-}^{\lambda},
$$

and from this formula we conclude immediately that

$$
\left(m^{2}+P \pm i 0\right)^{\lambda}=\left(m^{2}+P-i 0\right)^{\lambda}=\left(m^{2}+P\right)^{\lambda}
$$

when $\lambda=k$ positive integer. 
We observe that $\left(m^{2}+P \pm i 0\right)^{\lambda}$ are entire distributional functions of $\lambda$. This is the principal difference between the distributions, formally analogue $(P \pm i 0)^{\lambda}$ which have poles at the points $\lambda=-\frac{n}{2}-k, k=0,1,2, \ldots$

It can be proved ([4], page 573, formula (2.14) and page 575, formula (3.5)) that

$$
\left(m^{2}+P \pm i 0\right)^{-k}=P f\left(m^{2}+P\right)^{-k} \mp \frac{\pi i(-1)^{k-1}}{(k-1) !} \delta^{(k-1)}\left(m^{2}+P\right)
$$

where $P f$ means finite part or regular part of the Laurent expansion of $\left(m^{2}+P\right)_{+}^{\lambda}$ about $\lambda=-k$, namely

$$
P f\left(m^{2}+P\right)^{-k}=\lim _{\lambda \rightarrow-k} \frac{d}{d \lambda}\left[(\lambda+k)\left(m^{2}+P\right)_{+}^{\lambda}\right] .
$$

The formula (55) is a multidimensional analogue of the well-known unidimensional formula (see formula (46)).

By causal (anticausal) distribution, we mean distributions of the form $f_{a}\left(n^{2}+P \pm i 0\right)$ where $\left(m^{2}+P \pm i 0\right)^{\lambda}$ is defined by the formula (51).

On the other hand, from [7], page 6, formulae (20) and (21), we have

$$
\begin{aligned}
& \left|m^{2}+P\right|^{\lambda}=\left(m^{2}+P\right)_{+}^{\lambda}+\left(m^{2}+P\right)_{-}^{\lambda} \\
& {\left[\operatorname{sgn}\left(m^{2}+P\right)\right]\left|m^{2}+P\right|^{\lambda}=\left(m^{2}+P\right)_{+}^{\lambda}-\left(m^{2}+P\right)_{-}^{\lambda}}
\end{aligned}
$$

and from [4], page 566, we have

$$
\operatorname{Res}_{\lambda=-k}\left(m^{2}+P\right)_{+}^{\lambda}=\frac{(-1)^{k-1}}{(k-1) !} \delta^{(k-1)}\left(m^{2}+P\right)
$$

and

$$
\operatorname{Res}_{\lambda=-k}\left(m^{2}+P\right)_{-}^{\lambda}=\frac{1}{(k-1) !} \delta^{(k-1)}\left(m^{2}+P\right) .
$$

Therefore, from (52), (53), (57), (58) and taking into account (59) and (60) we have

$$
\begin{aligned}
\lim _{\alpha \rightarrow-k} R_{\alpha}^{+}\left(m^{2}+P\right) & =\delta^{(k)}\left(m^{2}+P\right), \\
\lim _{\alpha \rightarrow-k} R_{\alpha}^{-}\left(m^{2}+P\right) & =(-1)^{k} \delta^{(k)}\left(m^{2}+P\right), \\
\lim _{\alpha \rightarrow-2 k} Y_{\alpha}\left(m^{2}+P\right) & =\frac{(-1)^{k} k !}{(2 k) !} \delta^{(2 k)}\left(m^{2}+P\right),
\end{aligned}
$$

and

$$
\lim _{\alpha \rightarrow-2 k-1} Z_{\alpha}\left(m^{2}+P\right)=\frac{(-1)^{k+1} k !}{(2 k+1) !} \delta^{(2 k+1)}\left(m^{2}+P\right),
$$

where

$$
\begin{aligned}
& R_{\alpha}^{+}\left(m^{2}+P\right)=\frac{\left(m^{2}+P\right)_{+}^{\alpha-1}}{\Gamma(\alpha)}, \\
& R_{\alpha}^{-}\left(m^{2}+P\right)=\frac{\left(m^{2}+P\right)_{-}^{\alpha-1}}{\Gamma(\alpha)}
\end{aligned}
$$




$$
Y_{\alpha}\left(m^{2}+P\right)=\frac{\left|m^{2}+P\right|^{\alpha-1}}{\Gamma\left(\frac{\alpha}{2}\right)},
$$

and

$$
Z_{\alpha}\left(m^{2}+P\right)=\frac{\left|m^{2}+P\right|^{\alpha-1} \operatorname{sgn}\left(m^{2}+P\right)}{\Gamma\left(\frac{\alpha+1}{2}\right)} .
$$

In order to give a sense to formulae (61), (62), (63) and (64) (or "to regularize" them) we deal with multi-dimensional generalization obtained by means of a change of variable (see formula (7)).

Taking into account the formulae (8) and (9) we are going to obtain the Hankel distributional transform of the family of generalized functions defined by the formulae (65), (66), (67), (68) and (54).

In fact, from (8) and (9) we have

$$
\langle H\{\Phi(u(x)\}, \varphi\rangle=\langle H\{v(t)\}, \psi(s)\rangle
$$

where $\psi(s)$ is defined by the formula (10).

From (32) and (69) we have,

$$
\begin{aligned}
& \left\langle H\left\{R_{\alpha}^{+}\left(m^{2}+P\right)\right\}, \varphi\right\rangle=\left\langle H\left\{R_{\alpha}^{+}(t)\right\}, \psi(s)\right\rangle= \\
& =\frac{1}{2^{\frac{n}{2}-2 \alpha}}\left\langle R_{\frac{n}{2}-\alpha}^{+}(s), \psi(s)\right\rangle= \\
& =\frac{1}{2^{\frac{n}{2}-2 \alpha}} \frac{1}{\Gamma\left(\frac{n}{2}-\alpha\right)}\left\langle S_{+}^{\frac{n}{2}-\alpha-1}, \psi(s)\right\rangle,
\end{aligned}
$$

where $S_{+}^{\lambda}$ is defined in (16).

Taking into account (10) and (7), we have

$$
\left\langle S_{+}^{\frac{n}{2}-\alpha-1}, \psi(s)\right\rangle=\left\langle\left(m^{2}+Q\right)_{+}^{\frac{n}{2}-\alpha-1}, \varphi\right\rangle
$$

where $\quad Q=Q(y)=g_{1}^{2}+\cdots+y_{\mu}^{2}-y_{\mu+1}^{2}-\cdots-y_{\mu+\nu}^{2}, \backslash \quad \mu+\nu=d$ (dimension of the space), and $\backslash \quad\left(m^{2}+Q\right)_{+}^{\lambda}$ is defined in (52).

From (70) and (71) we have proved our first basic formula:

$$
H\left\{R_{\alpha}^{+}\left(m^{2}+P\right)\right\}=\frac{1}{2^{\frac{n}{2}-2 \alpha}} R_{\frac{n}{2}-\alpha}^{+}\left(m^{2}+Q\right) .
$$

Similary, from (34) and (69) we have

$$
\begin{aligned}
& \left\langle H\left\{R_{\alpha}^{-}\left(m^{2}+P\right)\right\}, \varphi\right\rangle=\left\langle H\left\{R_{\alpha}^{-}(t)\right\}, \psi(s)\right\rangle= \\
& =\frac{(-1)^{\frac{n}{2}}}{2^{\frac{n}{2}-2 \alpha} \Gamma\left(\frac{n}{2}-\alpha\right)}\left\langle S_{-}^{-\alpha+\frac{n-2}{2}}, \psi(s)\right\rangle,
\end{aligned}
$$

where $S_{-}^{\lambda}$ is defined in (17).

Taking into account (10) and (7), we have

$$
\left\langle S_{-}^{-\alpha+\frac{n-2}{2}}, \psi(s)\right\rangle=\left\langle\left(m^{2}+Q\right)_{-}^{\frac{n}{2}-\alpha-1}, \varphi\right\rangle,
$$


where $\left(m^{2}+Q\right)^{\lambda}$ is defined in $(53)$.

From (73) and (74) we have proved our second basic formula:

$$
H\left\{R_{\alpha}^{-}\left(m^{2}+P\right)\right\}=\frac{(-1)^{\frac{n}{2}}}{2^{\frac{n}{2}-2 \alpha}} R_{\frac{n}{2}-\alpha}^{-}\left(m^{2}+Q\right) .
$$

On the other hand, from (67), (36), (69) and taking into account the formulae (71) and (74) we have

$$
\begin{aligned}
& \left\langle H\left\{Y_{\alpha}\left(m^{2}+P\right)\right\}, \varphi\right\rangle=\left\langle H\left\{Y_{\alpha}(t), \psi(s)\right\}\right\rangle= \\
& =a(\alpha, n, \pi)\left[\left\langle R_{\frac{n}{2}-\alpha}^{+}(s)+(-1)^{\frac{n}{2}} R_{\frac{n}{2}-\alpha}^{-}(s), \psi(s)\right\rangle\right]= \\
& =a(\alpha, n, \pi)\left[\left\langle R_{\frac{n}{2}-\alpha}^{+}(s), \psi(s)\right\rangle+(-1)^{\frac{n}{2}}\left\langle R_{\frac{n}{2}-\alpha}^{-}(s), \psi(s)\langle]=\right.\right. \\
& =a(\alpha, n, \pi)\left[\left\langle R_{\frac{n}{2}-\alpha}^{+}\left(m^{2}+Q\right), \varphi\right\rangle+(-1)^{\frac{n}{2}}\left\langle R_{\frac{n}{2}-\alpha}^{-}\left(m^{2}+Q\right), \varphi\right\rangle\right]= \\
& =a(\alpha, n, \pi)\left[\left\langle R_{\frac{n}{2}-\alpha}^{+}\left(m^{2}+Q\right)+(-1)^{\frac{n}{2}} R_{\frac{n}{2}-\alpha}^{-}\left(m^{2}+Q\right), \varphi\right\rangle\right] ;
\end{aligned}
$$

where

$$
a(\alpha, n, \pi)=\frac{\Gamma\left(\frac{\alpha+1}{2}\right)}{2^{\frac{n}{2}-3 \alpha+1} \pi^{\frac{1}{2}}} .
$$

Therefore, from (76) we obtain the following formula:

$$
H\left\{\frac{\left|m^{2}+P\right|^{\alpha-1}}{\Gamma\left(\frac{\alpha}{2}\right)}\right\}=a(\alpha, n, \pi)\left[R_{\frac{n}{2}-\alpha}^{+}\left(m^{2}+Q\right)+(-1)^{\frac{n}{2}} R_{\frac{n}{2}-\alpha}^{-}\left(m^{2}+Q\right)\right],
$$

where $a(\alpha, n, \pi)$ is defined by the formula (77).

Similarly, from (68), (37) and taking into account the formulae (71) and (74), we have

$$
\begin{aligned}
& \left\langle H\left\{Z_{\alpha}\left(m^{2}+P\right)\right\}, \varphi\right\rangle=\left\langle H\left\{Z_{\alpha}(t)\right\}, \psi(s)\right\rangle= \\
& =b(\alpha, n, \pi)\left[\left\langle R_{\frac{n}{2}-\alpha}^{+}(s)-(-1)^{\frac{n}{2}} R_{\frac{n}{2}-\alpha}^{-}(s), \psi(s)\right\rangle\right]= \\
& =b(\alpha, n, \pi)\left[\left\langle R_{\frac{n}{2}-\alpha}^{+}(s), \psi(s)\right\rangle-(-1)^{\frac{n}{2}}\left\langle R_{\frac{n}{2}-\alpha}^{-}(s), \psi(s)\right\rangle\right]= \\
& =b(\alpha, n, \pi)\left[\left\langle R_{\frac{n}{2}-\alpha}^{+}\left(m^{2}+Q\right), \varphi\right\rangle-(-1)^{\frac{n}{2}}\left\langle R_{\frac{n}{2}-\alpha}^{-}\left(m^{2}+Q\right), \varphi\right\rangle\right]= \\
& =b(\alpha, n, \pi)\left[\left\langle R_{\frac{n}{2}-\alpha}^{+}\left(m^{2}+Q\right)-(-1)^{\frac{n}{2}} R_{\frac{n}{2}-\alpha}^{-}\left(m^{2}+Q\right), \varphi\right\rangle\right] ;
\end{aligned}
$$

where

$$
b(\alpha, n, \pi)=\frac{\Gamma\left(\frac{\alpha}{2}\right)}{2^{\frac{n}{2}-3 \alpha+1} \pi^{\frac{1}{2}}} .
$$

Therefore, from (79) we obtain the following formula:

$$
\begin{aligned}
& H\left\{\frac{\left|m^{2}+P\right|^{\alpha-1} \operatorname{sgn}\left(m^{2}+P\right)}{\Gamma\left(\frac{\alpha+1}{2}\right)}\right\}= \\
& =b(\alpha, n, \pi)\left[\left\langle R_{\frac{n}{2}-\alpha}^{+}\left(m^{2}+Q\right)-(-1)^{\frac{n}{2}} R_{\frac{n}{2}-\alpha}^{-}\left(m^{2}+Q\right)\right],\right.
\end{aligned}
$$

where $b(\alpha, n, \pi)$ is defined by the formula (80). 
Finally, to obtain the Hankel transform of $f_{\alpha}\left(m^{2}+P \pm i 0\right)$, we shall take into account the results (72) and (75).

In fact, for $n$ even, from (54) and taking into account the formulae (72) and (75) we have

$$
\begin{aligned}
& H\left\{f_{\alpha}\left(m^{2}+P \pm i 0\right)\right\}=\Gamma(\alpha)\left[H\left\{R_{\alpha}^{+}\left(m^{2}+P\right)\right\}+e^{ \pm(\alpha-1) \pi i}\right. \\
& \left.\cdot H\left\{R_{\alpha}^{-}\left(m^{2}+P\right)\right\}\right]=\frac{\Gamma(\alpha)}{2^{\frac{n}{2} 2 \alpha}} R_{\frac{n}{2}-\alpha}^{+}\left(m^{2}+Q\right)+ \\
& +\frac{e^{ \pm(\alpha-1) \pi i} \Gamma(\alpha)}{2^{\frac{n}{2}-2 \alpha}} R_{\frac{n}{2}-\alpha}^{-}\left(m^{2}+Q\right)= \\
& =\frac{\Gamma(\alpha)}{2^{\frac{n}{2}-2 \alpha} \Gamma\left(\frac{n}{2}-\alpha\right)}\left(m^{2}+Q \mp i 0\right)^{\frac{n}{2}-\alpha-1}= \\
& =\frac{\Gamma(\alpha)}{2^{\frac{n}{2}-2 \alpha} \Gamma\left(\frac{n}{2}-\alpha\right)} f_{\frac{n}{2}-\alpha}\left(m^{2}+Q \mp i 0\right)
\end{aligned}
$$

Therefore, from (82) we obtain the following formula:

$$
H\left\{\left(m^{2}+P \pm i 0\right)^{\alpha-1}\right\}=\frac{\Gamma(\alpha)}{2^{\frac{n}{2}-2 \alpha} \Gamma\left(\frac{n}{2}-\alpha\right)}\left(m^{2}+Q \mp i 0\right)^{\frac{n}{2}-\alpha-1},
$$

$\alpha \neq-k, k=0,1,2, \ldots$, and $n$ even. In particular, for the case $\alpha=-k, k=0,1,2, \ldots$, and taking into account the formula (61), from (72) we get the following result

$$
\begin{aligned}
& H\left\{\delta^{(k)}\left(m^{2}+P\right)\right\}=\frac{1}{2^{\frac{n}{2}+2 k}} R_{\frac{n}{2}+2 k}^{+}\left(m^{2}+Q\right)= \\
& =\frac{1}{2^{\frac{n}{2}-2 k} \Gamma\left(\frac{n}{2}+k\right)}\left(m^{2}+Q\right)_{+}^{\frac{n-2}{2}+k} .
\end{aligned}
$$

The formula (84) was proved in [5], page 3, formula (36).

We observe that if consider the following family generalized functions $N_{\alpha}\left(m^{2}+P \pm i 0\right)$ defined by the formula

$$
N_{\alpha}\left(m^{2}+P \pm i 0\right)=\frac{f_{\alpha}\left(m^{2}+P \pm i 0\right)}{\Gamma(\alpha)}
$$

where $f_{\alpha}\left(m^{2}+P \pm i 0\right)$ is defined by (54), and taking into account the formula (82), then the Hankel distributional transform of $N_{\alpha}\left(m^{2}+P \pm i 0\right)$ can be expressed by the formula

$$
H\left\{N_{\alpha}\left(m^{2}+P \pm i 0\right)\right\}=\frac{1}{2^{\frac{n}{2}-2 \alpha}} N_{\frac{n}{2}-\alpha}\left(m^{2}+Q \mp i 0\right)
$$

for $n$ even and $\alpha \neq-k, k=0,1,2, \ldots$

For the case $\alpha=-k$, taking into account the formulae ((61) and ((62), we have

$$
\lim _{\alpha \rightarrow-k} N_{\alpha}\left(m^{2}+P \pm i 0\right)=0
$$

where the limit is in the sense of generalized functions. 
M. AGUIRRE

\section{References}

[1] Schwartz, L. (1966) Théorie des distributions. Hermann, Paris.

[2] Leray, J. (1952) Hyperbolic Differential Equations. Mimeographed Lecture Notes, Inst. for Advanced Studies, Princeton.

[3] Gelfand, I.M.; Shilov, G.E. (1964) Generalized Functions. Vol I, Academic Press, New York.

[4] Bresters, D.W. (1968) "On distributions connected with quadratic forms", SIAM J. Appl. Math. 16: 563-581.

[5] Aguirre, M.A.; Trione, S.E. "The distributional Hankel transform of $\delta^{(k)}\left(m^{2}+P\right)$ ", Studies in Applied Mathematics 83: 111-121, Massachusetts Institute of Technology Publ., Elsevier Science Publ. Co. Inc.

[6] Trione, S.E. (1980) Distributional Products. Cursos de Matemática, N 3, Serie II, IAM-CONICET.

[7] Trione, S.E. (1987) "The multiplicative product between $P f\left(m^{2}+P\right)$ and $\delta^{(\gamma-1)}\left(m^{2}+\right.$ $P)$, and others", Trab. de Matemática, Serie I, N 130, IAM-CONICET.

[8] Bateman Manuscript (1953) Higher Trascendental Functions, Vol. I and II. McGrawHill, New York.

[9] Grandshtegn, I.S.; Ryzhik, I.M. Table of Integrals Series and Products. Corrected and enlarged edition. Academic Press Inc. 